\title{
MONTE CARLO DYNAMICS OF DIAMOND-LATTICE MULTICHAIN SYSTEMS
}

\author{
Andrzej Kolinski, * Jeffrey Skolnick ${ }^{\dagger}$ and Robert Yaris \\ Department of Chemistry, Washington University, St. Louis, MO
}

63130

\section{ABSTRACT}

We present preliminary results of Monte Carlo studies on the dynamics of multichain diamond-lattice systems at considerably greater densities than those done previously. Chain dynamics were simulated by a random sequence of three and four bond kink motions. The single bead autocorrelation function exhibits "slow" mode relaxation behavior with a $g(t) \propto t^{\beta}$. There is a smooth crossover from Rouse-like dynamics, $\beta=1 / 2$, at low density to smaller values of $\beta$ at higher density and $\beta=0$ at the glass transition density $\left(\phi_{G} \cong 0.92\right)$. The simulation provides a self-diffusion coefficient $D \propto \alpha^{-2}$, with $n$ the number of beads, in agreement with experiment. A phenomenologica $\bar{T}$ model, different from the widely accepted reptation picture, is proposed.

\section{INTRODUCTION}

The presently accepted model for polymer dynamics in a melt is the "reptation" model of de Gennes.1. The model assumes Rouse-like motion of the chain along a "tube" formed by entanglements with other chains. The tube renewal time is believed to be much longer than the relaxation time of the local "defects" along the chain. Within the reptation framework the self-diffusion coefficient (D) depends on chain length $(n)$ as $D \propto n^{-2}$, the melt viscosity $\eta \propto n^{-3}$ and the single bead autocorrelation function $g(t)$ should exhibit a "slow" relaxation mode giving a $g(t) \propto t 1 / 4$, connected with the reptation of the chain within the Gaussian tube.

Numerous Monte Carlo (MC) simulations $2-7$ have been performed to check the validity of reptation. To date no evidence for reptation has been found if all the chains are mobile, but reptation is observed when a single chain moves in a network of fixed obstacles. 2-7

The present simulations were performed on diamond lattice systems containing long chains packed at densities substantially higher than in previous papers 2,4 which employed a very similar model of the dynamics (see for example, ref. 2).

\section{MONTE CARLO MODEL}

Simulations were performed on $N$ chains containing $n$ beads confined to a tetrahedral lattice subject to multiple exclusion of lattice sites but no attractive interactions (an athermal system). Periodic boundary conditions were employed on the MC box of volume $\underline{L}^{3}$, where $\underline{L}$

\footnotetext{
*permanent address: Department of Chemistry, University of Warsaw, 02-093 Warsaw, Poland

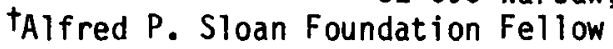


was considerably greater than the root-mean-square radius of gyration of the polymer chains. We define the polymer volume fraction $\phi$ as the fraction of occupied lattice sites. Initial configurations were obtained from very carefully equilibrated systems.8 A random sequence of 3 - and 4-bond conformational jumps 2 was used to simulate the dynamics and the time unit was assumed to be that required for one (on the average) attempt of these micromodifications per polymer bead.

\section{RESULTS AND DISCUSSION}

We computed various autocorrelation functions for model polymers, e.g. the single bead autocorrelation function $g(t)=\left\langle\left(r_{j}(t)-r_{j}(0)\right\rangle^{2}\right\rangle$ where $r_{i}(t)$ is the coordinate of polymer bead $i$ at time $t$, and the averaging was performed on the $n-20$ middle units of all $N$ chains. For the center of mass autocorrelation function, $g_{\mathrm{cm}}(t)$, all the bead coordinates were counted. Figures 1 and 2 show the behavior of $g(t)$ for various chain lengths (at constant $\phi$ ) and densities $\phi$ (at constant n) respectively. Inspection of Fig. 1 suggests that the "slow" diffusional regime (where $g(t) \propto t 0.33$ at this particular concentration) is rather insensitive to chain length and seems to be connected with the local relaxation of the subchains. Figure 2 shows the crossover from Rouse-like motion $(g(t) \propto t 1 / 2)$ to very slow diffusion regime where $g(t) \propto t^{\beta}$, where $\beta$ is concentration dependent.

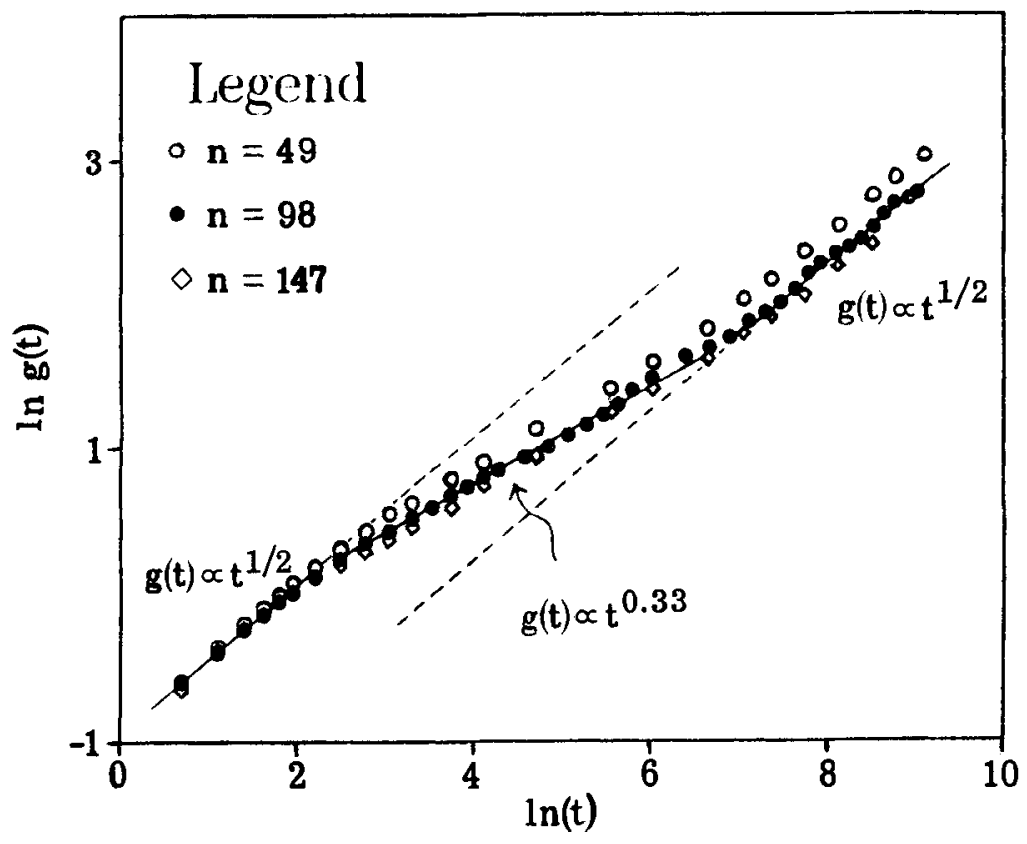

Figure 1

Fig. 1. Log-log plots of $g(t)$ versus time for various chain lengths. $\dot{\phi}=0.75$. 


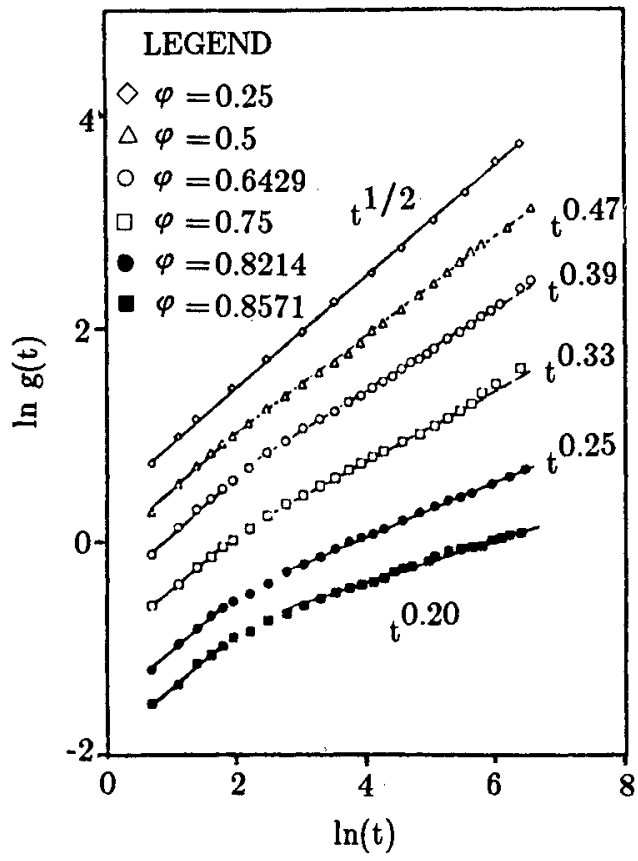

Figure 2

Log-log plots of $g(t)$ versus time for various volume fractions of polymers. $\underline{n}=98$.

The plateau $(\beta=0)$ regime in $g(t)$, where there are no long distance motions, can be achieved either by an increase in concentration or by the exclusion of 4-bond motions (the source of new orientations of the chain segments) at intermediate concentration. (However, we point out that the exponent $B$ is rather insensitive to the particular values of the a priori probabilities of 3- and 4-bond jumps employed provided that both are considerably greater than zero.) In Fig. 3 , we extrapolate the ratio of the fraction of four to three

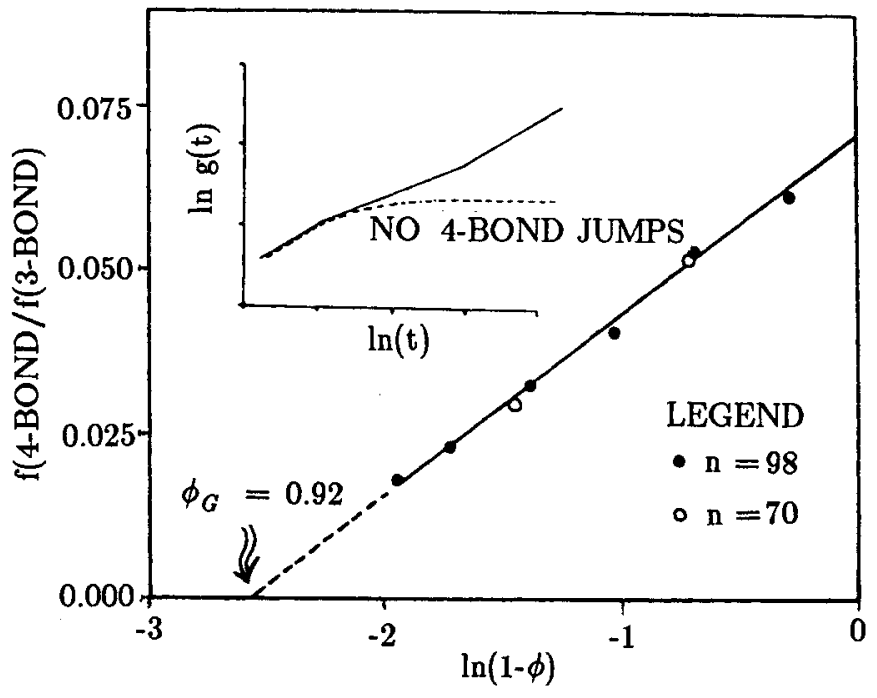

Figure 3

$f(4-$ bond $) / f$ (3-bond), plotted against in $(1-\phi)$ with $\underline{n}=98$. The subplot shows the difference between liquid-like and glass-like behavior of $g(t)$ at $\phi=$ 0.75 as an example (glass-like motion results from a priori exclusion of 4-bond jumps). 
bond motions ( $f(4-$ bond $) / f(3-$ bond $) \rightarrow z e r o$. The predicted glasstransition density $\phi_{G}=0.92 \pm 0.01$ is in agreement with that previously found. 9

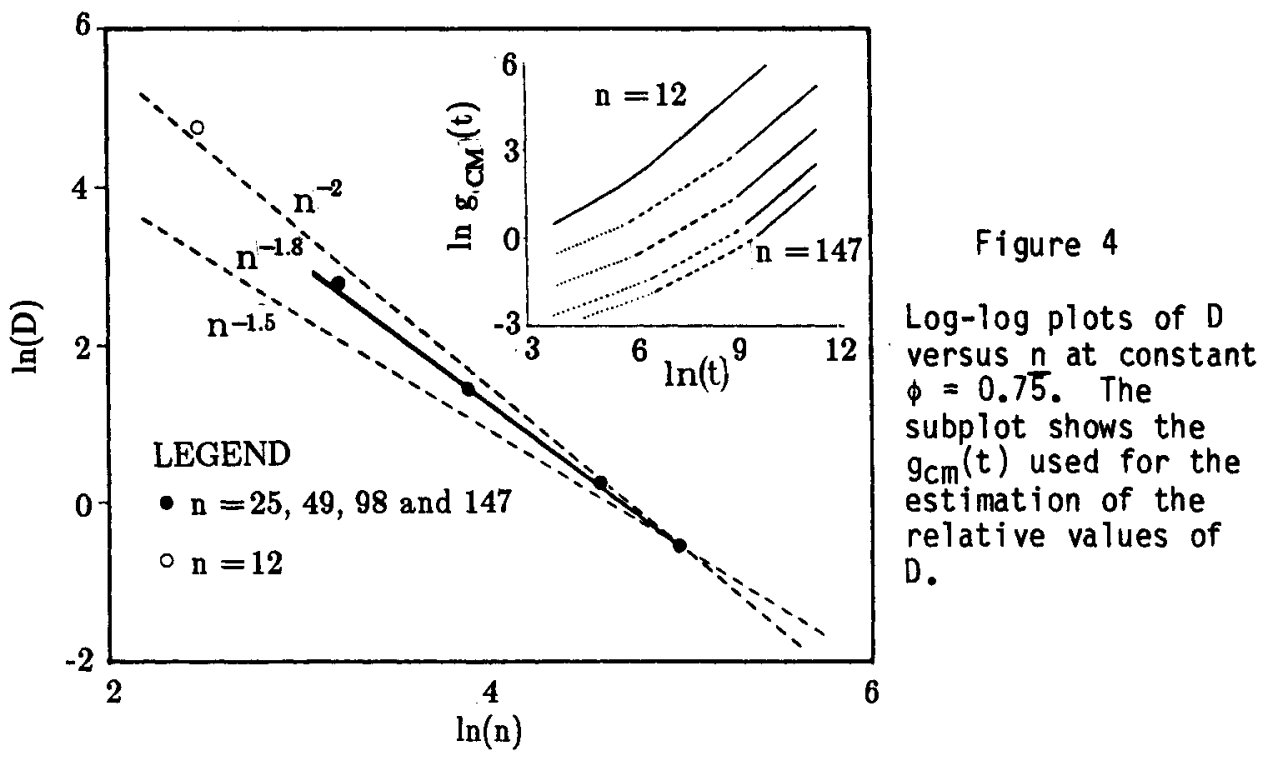

From the long time value of $g_{\mathrm{cm}}(t)$ shown in Fig. 4, we estimated the self-diffusion coefficient of the polymer and obtained $D \propto n^{-\alpha}$ with $\alpha=1.8 \pm 0.2$, in qualitative agreement with experiment. 10

The analysis of other correlation functions ${ }^{8}$ (such as the diffusion of holes or kinks) shows that the local conformational

"defects" diffuse faster transverse to the local chain axis than down the chain contour. The defects diffuse like particles in a collection of boxes. At short times there is free diffusion within a box, followed by a pausing time that reflects the wait before hopping to the next box. Finally, at longer times, jumps between the boxes dominate and diffusive behavior reemerges. The net result of this transverse defect motion is that the dynamics of the melt resembles that of a bowl of amoebas. This is graphically depicted in Fig. 5 where we show a representative example of a single chain in the melt whose initial and final states are separated by 45,000 time steps. No evidence for reptation is seen.

\section{CONCLUSION}

The relaxation process of polymers in a melt is seen to be a cooperative phenomenon involving amoeboid-like motion of blobs. Our present view on long distance polymer motion in a melt is that it is intrinsically a many-body problem requiring back flow between chains to maintain constant density. 


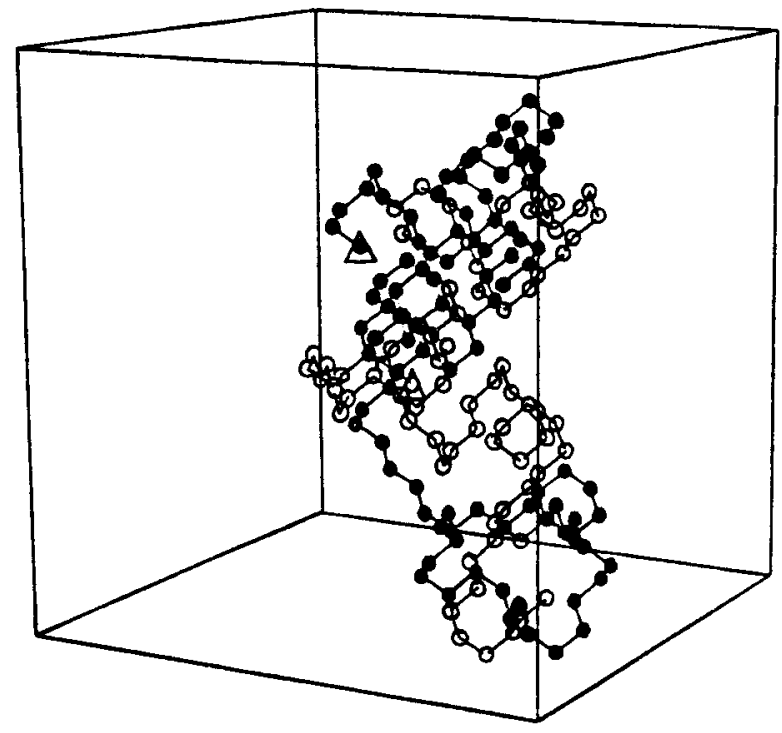

Figure 5

Two superimposed snapshots of a polymer chain separated by $\mathrm{T}=$ 45,000 time steps. $T$ is sufficently large so that $g(t)$ is on the order of the radius of gyration. Note that some parts of the chain have remained stationary. $\phi=0.75$ and $\underline{n}=98$.

Acknowledgement. We are deeply grateful to Professor Bruno Zimm for pointing out the possibility of a glass transition in these systems. This work was supported in part by the National Science Foundation Polymers Program and the Petroleum Research Fund.

\section{REFERENCES}

1. P.G. de Gennes, "Scaling Concepts in Polymer Physics", Corne11 University Press (1979).

2. K. Kremer, Macromolecules 16, 1632 (1983).

3. D. Richter, A. Baumgartner, K. Binder, B. Ewen and J.B. Hayter, Phys. Rev. Lett. 47, 109 (1981).

4. K. Kremer, Phys. Rev. Lett. 51, 1923 (1983).

5. K.E. Evans, S.F. Edwards, J. Chem. Soc., Faraday Trans. 2, 77, 1891 (1981).

6. K. Kremer, K. Binder, J. Chem. Phys. 81, 6381 (1984).

7. M. Bishop, D. Ceperley, H.L. Frisch, M.H. Kalos, J. Chem. Phys. 76, 1557 (1982).

8. A. Kolinski, J. Skolnick, R. Yaris (unpublished).

9. R.D. Batie, J.L. Viovy and L. Monnerie, J. Chem. Phys, 81, 567 (1984).

10. J.D. Ferry, "Viscoelastic Properties of Polymers", Wiley, NY 1970. 